\title{
HP1 $y$ expression is elevated in prostate cancer and is superior to Gleason score as a predictor of biochemical recurrence after radical prostatectomy
}

\author{
Jon Slezak ${ }^{1 \dagger}$, Matthew Truong ${ }^{1 \dagger}$, Wei Huang ${ }^{2}$ and David Jarrard ${ }^{3 *}$
}

\begin{abstract}
Background: Aberrant chromatin structure in cancer cells results from altered proteins involved in its packaging. Heterochromatin protein 1 gamma (HP1 $\mathrm{y}$ ) is a non-histone heterochromatic protein that functions to maintain chromatin stability and is important in embryonic development. Given an interest in the role developmental genes play in cancer, we investigated HP1 $y$ expression in prostate cancer (PCa) and its prognostic associations.

Methods: Tissue microarrays consisting of benign $(N=96)$, localized cancer $(N=146)$, metastatic PCa $(N=44)$, and HGPIN ( $=50$ ) were immunoflourescently stained for HP1Y and Ki-67. Using a novel, automated quantitative imaging system, VECTRA ${ }^{\text {TM }}$, epithelial staining in both the nucleus and cytoplasm was quantified and compared against clinicopathologic variables.

Results: HP1y is significantly elevated in HGPIN (80\%), localized PCa (76\%), and metastatic PCa (98\%) compared to benign tissues from both the nuclear and cytoplasmic compartments $(P<0.0001)$. Increased nuclear and total HP1 $\gamma$ expression was associated with Gleason score $(P=0.02$ and $P=0.04$ respectively). Given known binding to the C-terminus of Ki-67, a co-expression analysis was performed that revealed a correlation between nuclear and cytoplasmic HP1Y and Ki-67 (Pearson Coefficient 0.321 and 0.562 respectively, $P<0.0001$ ). Cox survival analysis demonstrated that cytoplasmic HP1y expression was an independent prognostic marker and out-performed pathological Gleason score for predicting PSA-recurrence after radical prostatectomy.

Conclusions: In this first detailed analysis of HP1y expression in cancer, VECTRA ${ }^{\text {TM }}$ demonstrates compartmentalized and total HP1Y protein expression is increased in PCa and that expression correlates with clinical outcomes better than Gleason score. Given the critical role HP1Y plays in chromatin organization and gene expression, it represents a novel prognostic and therapeutic target.
\end{abstract}

Keywords: Prostate cancer, HP1Y, Prognosis, PSA-Recurrence, Ki-67, Tissue microarray

\section{Background}

Prostate cancer $(\mathrm{PCa})$ is the second most common cancer in men and will account for approximately 28,170 deaths in 2012 [1]. The behavior of some cancers can be variable despite Gleason score and other clinicopathologic factors. Recent efforts have focused on developing biomarkers that provide clinicians with the improved ability to identify

\footnotetext{
*Correspondence: Jarrard@urology.wisc.edu

'Equal contributors

${ }^{3}$ Department of Urology, University of Wisconsin-Madison, Madison, WI 53705, USA

Full list of author information is available at the end of the article
}

clinically significant cancers and aid in treatment decisions. Genes important in embryogenesis frequently play a role in cancer [2]. One such gene, heterochromatin pro-

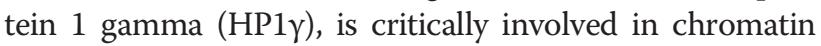
packaging [3] and demonstrates altered expression during development and cell differentiation [4-6].

HP1 $\gamma$, along with HP1 $\alpha$ and HP1 $\beta$, belong to a family of heterochromatin proteins with stabilizing functions. The structure of HP1 contains an evolutionarily conserved chromodomain that binds to a methylated lysine on histone $\mathrm{H}_{3}$ (H3K9me) [7]. This binding epigenetically

\section{Biomed Central}

(c) 2013 Slezak et al.; licensee BioMed Central Ltd. This is an Open Access article distributed under the terms of the Creative Commons Attribution License (http://creativecommons.org/licenses/by/2.0), which permits unrestricted use, distribution, and reproduction in any medium, provided the original work is properly cited. 
marks regions of silenced or reduced gene expression [8]. HP1 stabilizes telomeric and centromeric heterochromatin structure and facilitates DNA repair after disruption or damage $[9,10]$. This chromatin repair function is important to maintain information encoded in the epigenetic histone code [9]. The HP1 isoforms appear to have differential functions. HP1 $\gamma$ has the ability to regulate both heterochromatin and euchromatin structure, while the other isoforms are localized only on heterochromatin.

Decreased expression of HP1 $\gamma$ occurs during cell differentiation which is unique to this isoform compared to $\mathrm{HP} 1 \alpha$ and $\beta$ [6]. In differentiated human tissues levels of

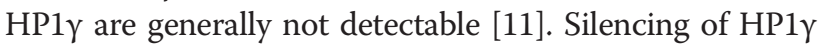
has functional consequences. In selected cancer cell lines, growth is inhibited. In a recent screen of cancer tissues, HP1 $\gamma$ protein appeared to be overexpressed in samples of lung, breast, colon and esophageal [5]. Although decreased levels of its isoform HP1 $\alpha$ were found in metastatic breast cancer compared to localized [12], $\mathrm{HP} 1 \gamma$ has not been previously evaluated as a marker for cancer progression.

Herein, we identify a novel overexpression of $\mathrm{HP} 1 \gamma$ in PCa tissues using a tissue microarray (TMA) and VECTRA $^{\mathrm{TM}}$ image acquisition technology. This quantitative tool permits localization of expression to nuclear versus cytoplasmic compartments. We report that nuclear and cytoplasmic $\mathrm{HP} 1 \gamma$ is progressively increased in HGPIN, $\mathrm{PCa}$, and metastatic PCa cores compared to benign tissue. Furthermore, increased $\mathrm{HP} 1 \gamma$ is correlated with Ki-67, a protein known to complex with other heterochromatin family proteins [13]. Finally, we report a novel prognostic role for cytoplasmic $\mathrm{HP} 1 \gamma$ in independently predicting PSA recurrence following prostatectomy. The use of highthroughput imaging technologies such as VECTRA allows for quantitative compartmentalization of marker expression to predict cancer outcomes and lends further insight into tumor biology.

\section{Methods}

\section{Tissue microarray}

The University of Wisconsin Institutional Review Board (IRB) provides ethical insight to clinical projects and reviews all human research protocols in accordance with federal regulations, state laws, and local and University policies. FFPE-patient tissues were obtained from the University of Wisconsin Department of Pathology and Laboratory Medicine under IRB approval. A tissue microarray was constructed using tissues from 64 PCa patients (mean age, 62.8 years) collected from 1995 to 2006. The mean follow-up period for this cohort was 9.6 years. The TMA consists of 336 duplicate cores from different disease groups: 43 localized PCa (pT2), 30 aggressive PCa (pT3 and 4), 21 metastatic $\mathrm{PCa}, 23$ high grade intraepithelial neoplasia (HGPIN) (tissue from HGPIN tissue blocks of some of the PCa patients of this cohort) and 48 benign prostate tissues (BPT) (from the non-tumor blocks of some of the cancer patients in this cohort).

\section{Staining}

Slide preparation and antigen retrieval were conducted as previously described [14]. Briefly, the TMA slides were taken through routine deparaffinization and rehydration, pretreated with endogenous peroxidase block and retrieval buffer. Slides were then rinsed with $\mathrm{dH} 2 \mathrm{O}$, then Tris Buffered Saline (TBS), then TBS with Tween (TBST), followed by protein blocking at room temperature. E-cadherin antibodies (Cell Signaling Technology, Beverly, MA) were used to define the epithelial compartment for better tissue segmentation. Slides were then stained with antibodies against HP1Y (EMD Millipore, Bilerica, MA) [15] and Ki-67 (Abcam, Cambridge, MA).

\section{Image analysis}

For automated image acquisition and analysis, the stained slides were loaded onto the slide scanner. Slides were scanned as previously described [14]. Cores with $<5 \%$ epithelial component or loss of tissue were excluded from the analysis. Nuance system and inForm $1.2^{\mathrm{TM}}$ software (Caliper Life Sciences, Hopkinton, MA) were used to for building spectral libraries on a per-cell basis for $\mathrm{HP} 1 \gamma$ and Ki-67 target signals according to manufacturer's protocols. This system allows automated quantitation of fluorescent staining on a per-cell basis and selection of cellular subsets (nucleus versus cytoplasmic) for analysis of target signals.

\section{Statistical analysis}

Nuclear and cytoplasmic expression of individual cores of various prostate tissues (benign, HGPIN, PCa, metastatic PCa) was statistically compared using the t-test. Correlation analysis was used to assess the relationship between $\mathrm{HP} 1 \gamma$ and Ki-67 expression in each compartment. We then assessed the relationship between $\mathrm{HP} 1 \gamma$ expression and patient clinicopathologic features using t-tests and analysis of variance (ANOVA). For this analysis, multiple PCa cores obtained from the same patient were first averaged, providing a more precise estimation of core expression in each biological replicate. HP1 $\gamma$ expression was then compared with clinicopathologic information (PSA, Gleason, pT stage, tumor volume, SV involvement, margins, extracapsular extension, and evidence of metastasis) collected from the 64 patients included in this study. Univariate and multivariate Cox regression analyses were used to assess whether nuclear and cytoplasmic $\mathrm{HP} 1 \gamma$ (as continuous variables) predicted biochemical recurrence following prostatectomy. Statistically significant variables from univariate Cox analysis were then entered into a multivariate Cox regression model. Variables that were not independent predictors of recurrence were removed using backward selection $(P<0.05)$. SPSS Version 
20.0 (IBM, Armonk, New York) was used to perform statistical analyses. All tests were two-tailed and a $P$ value $<0.05$ was considered statistically significant.

\section{Results}

HP1 1 expression in prostate core samples is elevated in PCa, HGPIN, and metastatic PCa

We measured HP1 $\gamma$ expression on TMAs using VECTRA $^{\mathrm{mm}}$ quantitative immunofluorescent analysis. Expression was measured in both the nuclear and cytoplasmic compartments of epithelial and stromal cells. In normal prostate tissues, $\mathrm{HP} 1 \gamma$ was expressed at low levels. Total expression of $\mathrm{HP} 1 \gamma$ was significantly increased in HGPIN and PCa, and was highest in metastatic tumors compared to benign samples (Figure 1). In the epithelial compartment, total, nuclear, and cytoplasmic expression were increased in $\mathrm{PCa}$ specimens compared to benign (Figure 2A-C) ( $P$-values <0.0001). Nuclear levels of HP1 $\gamma$ were 2-3 fold higher than cytoplasmic staining for all benign and cancer samples.

To define overexpression, ROC curve analysis was performed using total $\mathrm{HP} 1 \gamma$ expression to identify the optimum cut point that maximized the sum of the sensitivity and specificity for discriminating between benign versus cancer cores. ROC analysis demonstrated excellent discrimination between benign versus cancer (AUC = $0.796, P<0.0001$ ) with a sensitivity and specificity of $76.2 \%$ and $73.5 \%$, respectively (Figure $2 \mathrm{D}$ ). Using the optimum cut-off of 9.095 to define overexpression, 109/ 143 (76.2\%) of localized PCa cores, 40/50 (80\%) of HGPIN cores, and 40/41 (97.6\%) of metastatic PCa cores demonstrated overexpression of total $\mathrm{HP} 1 \gamma$ compared to only 23/88 (26.1\%) of benign cores.

\section{Ki-67 expression correlates with increased HP1Y expression in $P C a$}

$\mathrm{Ki}-67$ is a known marker of cell proliferation that has been demonstrated to complex with HP1 $\gamma$ on heterochromatin $[13,16,17]$. We assessed the correlation between HP1 $\gamma$ and Ki-67 on a per core basis. Within nuclear and cytoplasmic compartments there was a significant correlation between nuclear $\mathrm{Ki}-67$ and $\mathrm{HP} 1 \gamma$ expression (Figure 3A-C). The strength of the correlation was stronger for cytoplasmic than nuclear expression (Pearson Coefficients 0.562 and 0.321 , respectively, $P<0.0001)$.

\section{HP1Y expression is associated with Gleason score and pT category}

A series of clinicopathologic patient variables including seminal vesicle involvement, tumor volume, Gleason
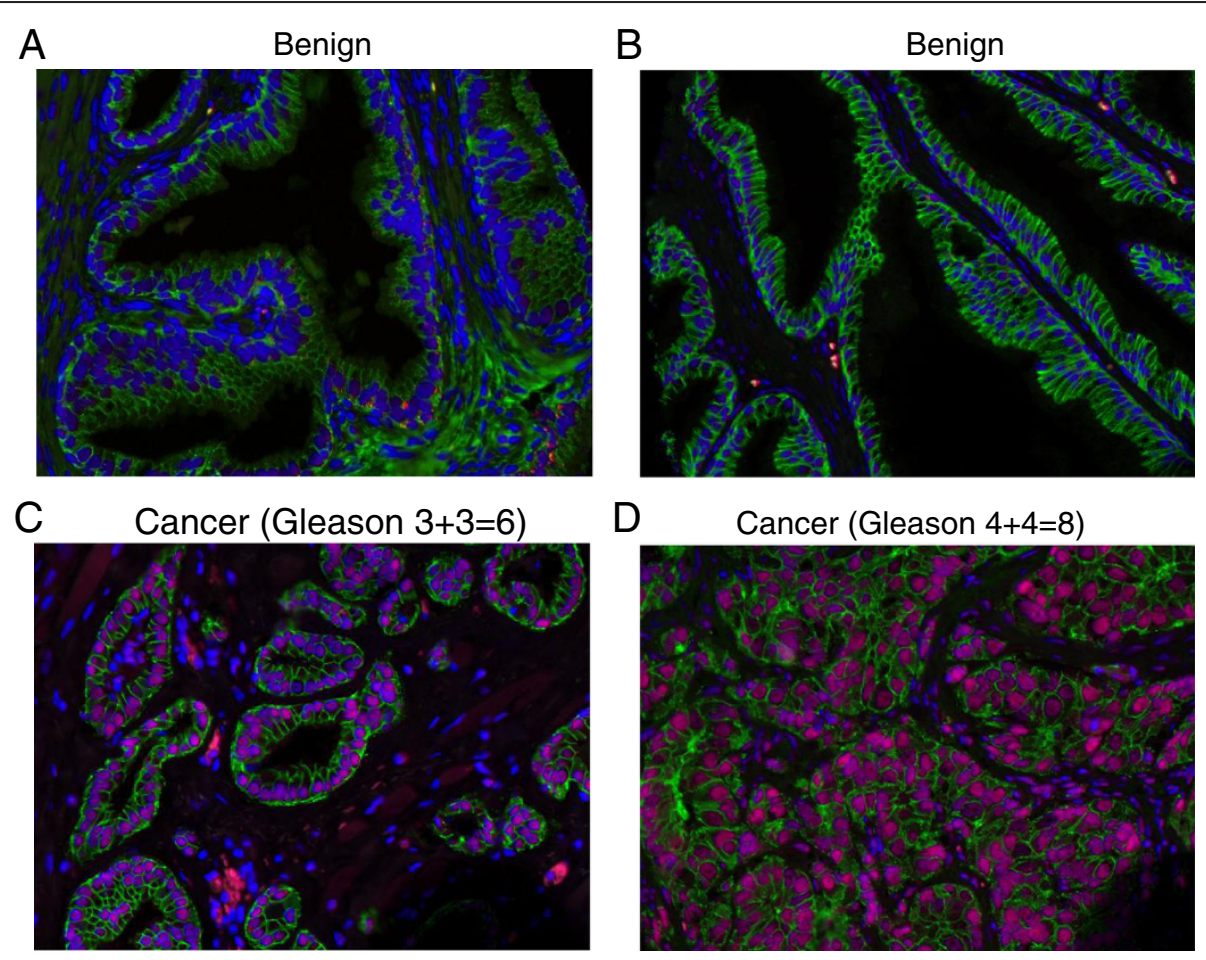

Figure $1 \mathrm{HP} 1 \gamma$ expression in human prostate cancer is increased compared to benign normal prostate tissue. Tissue microarrays (336 cores) containing benign (A and B), HGPIN, low grade (C), high grade (D) and metastatic PCa were immunoflourescently stained for HP1Y (red). Ecadherin (green) labels epithelial cells and DAPI (blue) the cell nuclei. Increased staining is noted in PCa samples with nuclear expression greater than cytoplasmic. Low levels of HP1Y expression were infrequently observed in benign epithelial cells. In contrast, HP1 $\gamma$ was uniformly upregulated in the majority of prostate cancer cells. 

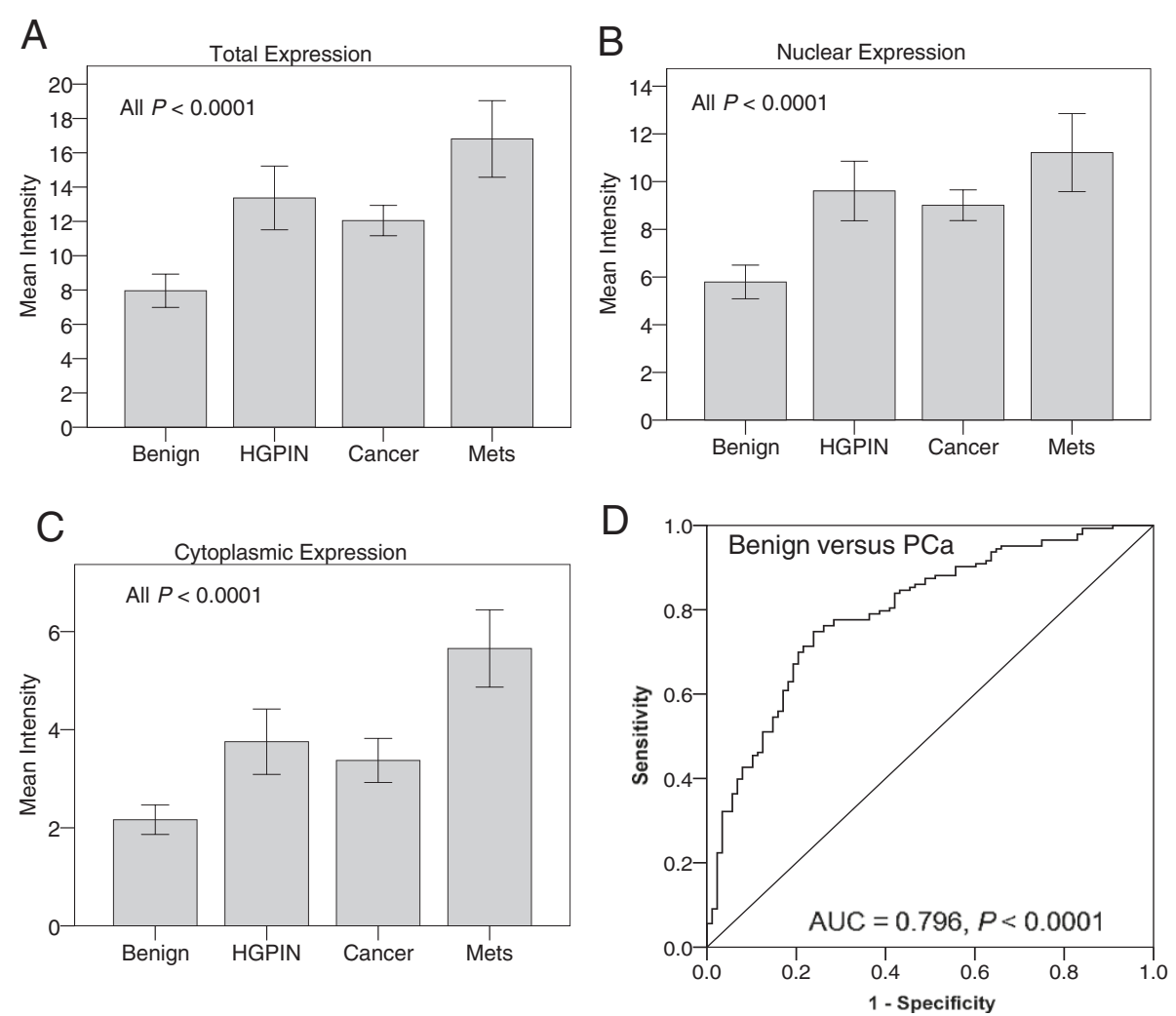

Figure 2 Quantitative analysis of HP1 $\gamma$ expression in human prostate cancer. VECTRA ${ }^{\text {TM }}$ technology was used for automated image attainment and analysis per the manufacturer's protocol. Both the nuclear and cytoplasmic compartments were compared. Cell intensities were averaged on a per cell basis for each core to determine the mean intensity of nuclear, cytoplasmic and total HP1y expression. Mean intensity of expression of HP1y was then compared for benign, HGPIN, cancer and metastasis core samples with 95\% confidence intervals. HP1y expression in HGPIN, cancer ( $\mathrm{N}=146$ cores), and metastasis is significantly greater than benign ( $\mathrm{N}=96$ cores) for total $(\mathbf{A})$, nuclear $(\mathbf{B})$, and cytoplasmic (C) expression with $P$ values $<0.0001$. Nuclear expression of the protein was $\sim 2.5$ fold greater than cytoplasmic expression. D) ROC curve analysis was used to determine the optimum cut-off for defining overexpression. Total HP1 $\gamma$ expression demonstrated excellent discrimination between benign and cancer (AUC $=0.796, P<0.0001$ ). A cut-off of 9.095 maximized the sensitivity $(76.2)$ and specificity $(73.5 \%)$ and was used to define overexpression.

score, margins, the presence of metastases, and extracapsular extension were compared to $\mathrm{HP} 1 \gamma$ nuclear and cytoplasmic expression. Using $\mathrm{HP} 1 \gamma$ expression as a continuous variable, we did not find an association of the majority of these variables with $\mathrm{HP} 1 \gamma$ expression (Table 1). However, the analysis did find that Gleason score was associated with nuclear and total expression ( $P=0.018$ and $P=0.042$, respectively). In summary, HP1 $\gamma$ expression does not appear to be tightly associated with many standard clinicopathologic variables.

\section{HP1Y expression predicts PSA recurrence}

Univariate Cox survival analysis demonstrated that Gleason score, stage (pT), seminal vesicle involvement, metastasis, and cytoplasmic HP1 $\gamma$ expression predicted biochemical recurrence $(P<0.05)$ (Table 2$)$. Both nuclear and cytoplasmic $\mathrm{HP \gamma}$ expression levels were analyzed as continuous variables. Furthermore, using multivariate Cox regression, we demonstrated that only the presence of metastasis and cytoplasmic $\mathrm{HP} \gamma$ were independent predictors of biochemical recurrence following prostatectomy $(P=$ 0.014 and $P=0.038$, respectively) (Table 3 ). Gleason score was no longer a statistically significant predictor of biochemical recurrence on multivariate analysis $(P>0.05)$. To verify that cytoplasmic $\mathrm{Hp} 1 \gamma$ was a strong predictor of recurrence than Gleason score, we performed a Kaplan Meier analysis of Gleason score alone (Figure 4A) versus each Gleason score stratified by high versus low cytoplasmic HP1 $\gamma$ expression (Figure 4B). Use of $\mathrm{HP} 1 \gamma$ allowed excellent separation of Gleason 7 patients into high (65\%) versus low (15\%) probability of biochemical recurrence. Furthermore, high $\mathrm{HP} 1 \gamma$ expression correctly predicted recurrence in 10/11 (91\%) of patients. However, with the Gleason system, having high-grade disease (Gleason 8 or 9) was only able to correctly identify 6/12 (50\%) cases of recurrence. 

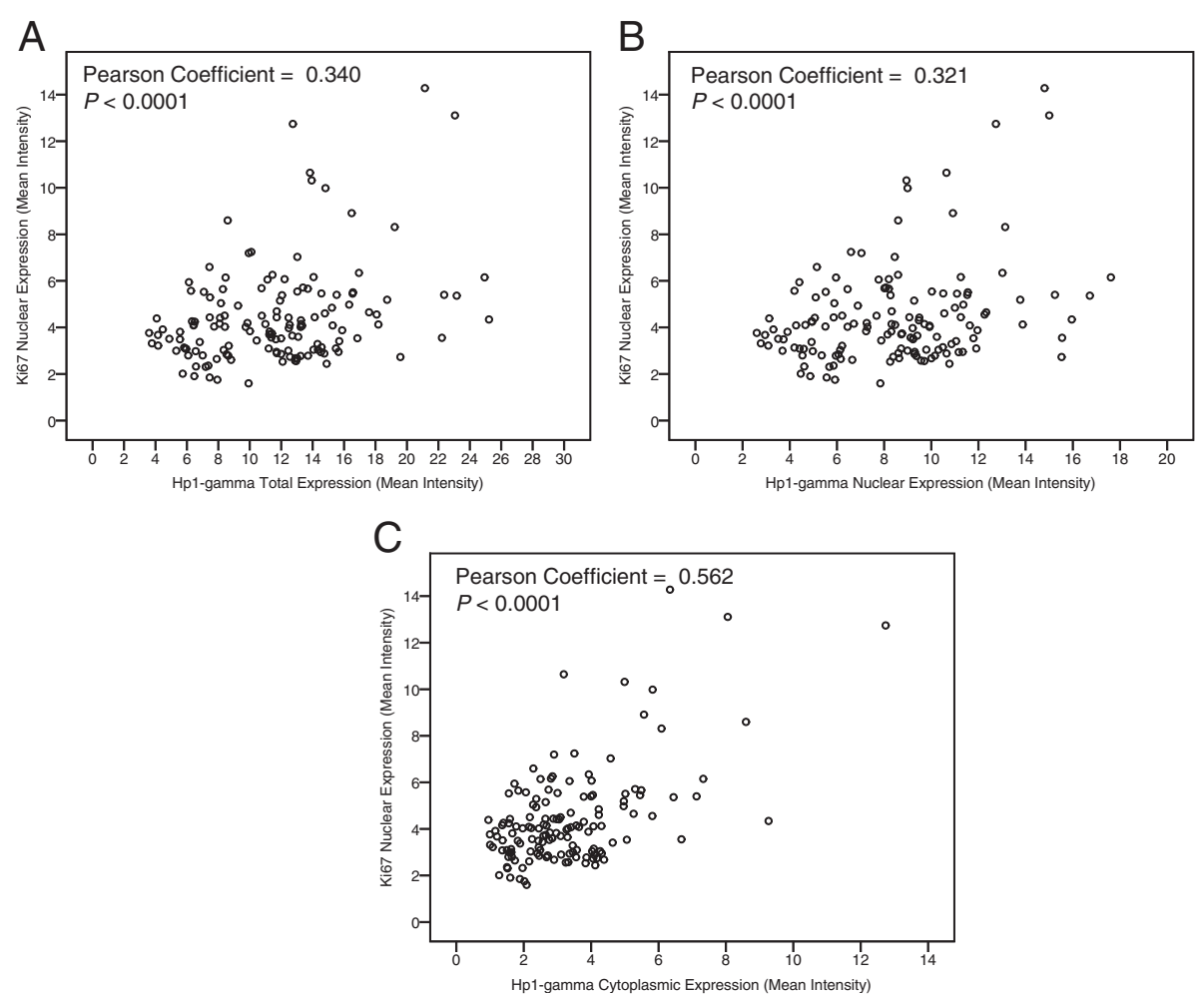

Figure $3 \mathrm{HP} 1 \gamma$ expression correlates with Ki-67. VECTRA ${ }^{\mathrm{TM}}$ technology was used for automated image attainment and analysis per the manufacturer's protocol to assess immunoflourescent staining of Ki-67 and HP1 $ү$. Pearson correlation coefficients yielded significant relationships between mean intensity expression of nuclear Ki-67 and $\mathbf{A})$ total $(P=0.34)$, B) nuclear $(P=0.32)$, and $\mathbf{C})$ cytoplasmic $(P=0.562) \mathrm{HP} 1 \gamma$. All $P$ values $<0.0001$.

\section{Discussion}

Patients and clinicians are in need of more accurate biomarkers to predict the prognosis of prostate cancer, especially for intermediate grade tumors [18]. Few markers have been reported that reliably predict treatment failure (e.g. PSA recurrence after surgery). Altered nuclear shape and size are a hallmark of cancer and reflect changes in chromatin structure. Image analyses of heterochromatin content and nuclear shape have been reported to improve the prediction of $\mathrm{PCa}$ prognoses [19]. HP1 $\gamma$ is a member of the HPI family of proteins involved in chromatin packaging and gene regulation. The current study represents the first in depth analysis of $\mathrm{HP} 1 \gamma$ expression for any cancer. We observe an increase in $\mathrm{HP} 1 \gamma$ expression in the majority of prostate cancers. Furthermore, by analyzing staining in distinct cellular compartments in a cohort of patients with extended follow-up, we find a role for the expression of this gene in predicting treatment failure.

A unique strength of this study lies in our use of the newly validated VECTRA ${ }^{\mathrm{m}}$ imaging technology. The platform merges automated slide scanning, multispectral imaging technology, and pattern recognition software into a system for biomarker analysis. VECTRA ${ }^{\mathrm{TM}}$ has many advantages over other imaging systems for biomarker quantitation [14]. VECTRA ${ }^{\mathrm{sm}}$ allows researchers to objectively analyze expression within separate epithelial and stromal tissue compartments. Nuclear and cytoplasmic staining within these compartments is concurrently assessed and multiple markers may be addressed simultaneously. The system is highly automated and uses pattern recognition to measure expression on a per-cell, core, or compartment basis on TMAs. The algorithm designed to segment tissue compartments by our genitourinary pathologist [WH] had a $97 \%$ rate of acceptable tissue segmentation. In the current analysis, VECTRA $^{\mathrm{Tm}}$ permitted a reproducible assessment of $\mathrm{HP} 1 \gamma$, and its association with Ki-67, in multiple cell compartments for a large cohort of patients.

HP1 $\gamma$ and the other HP1 family members have been primarily studied during embryogenesis and development, including that of the prostate [4]. Expression of $\mathrm{HP} 1 \gamma$ was noted in the fetal prostate at 14 and 24 weeks of gestation. This is in contrast to HP1 $\alpha$ which is not expressed at those developmental stages [4]. Decreased expression of $\mathrm{HP} 1 \gamma$ is required for normal cellular differentiation [5,6]. Our study demonstrates that expression of HP1 $\gamma$ occurs in $98 \%$ of metastatic and $76 \%$ of localized PCa tumors compared to $26 \%$ of benign tissues. This cut-off was determined by ROC analysis to 
Table 1 Association of $\mathrm{Hp} 1 \mathrm{Y}$ expression with patient pathological features

\begin{tabular}{|c|c|c|c|c|c|c|c|}
\hline \multirow{2}{*}{ Variable } & \multirow{2}{*}{ Number } & \multicolumn{2}{|l|}{ Nucleus } & \multicolumn{2}{|l|}{ Cytoplasm } & \multicolumn{2}{|l|}{ Total } \\
\hline & & Mean intensity (SD) & $\mathrm{p}$-value & Mean intensity (SD) & p-value & Mean intensity (SD) & $\mathrm{p}$-value \\
\hline \multicolumn{8}{|l|}{ Gleason } \\
\hline $3+3$ or $3+4$ & 47 & $8.77(2.5)$ & 0.018 & $3.02(0.8)$ & 0.095 & $11.8(3.2)$ & 0.042 \\
\hline $4+3$ or $4+4$ or $4+5$ & 12 & $10.74(2.5)$ & & $4.1(2.0)$ & & $14.9(4.5)$ & \\
\hline \multicolumn{8}{|l|}{ Stage } \\
\hline $\mathrm{T} 2$ & 40 & $8.62(2.7)$ & 0.238 & $2.86(0.9)$ & 0.039 & $11.48(3.4)$ & 0.12 \\
\hline T3 & 12 & $9.46(1.6)$ & & $3.47(0.7)$ & & $12.92(2.2)$ & \\
\hline T4 & 11 & $10.0(2.9)$ & & $3.77(2.0)$ & & $13.78(4.7)$ & \\
\hline \multicolumn{8}{|l|}{ Tumor Volume } \\
\hline$<5$ & 7 & $9.59(1.8)$ & 0.776 & $3.07(0.8)$ & 0.156 & $12.67(2.4)$ & 0.879 \\
\hline $5-20$ & 36 & $8.84(2.9)$ & & $3.06(1.4)$ & & $11.91(4.0)$ & \\
\hline$>20$ & 20 & $9.09(2.4)$ & & $4.01(2.5)$ & & $12.03(3.1)$ & \\
\hline \multicolumn{8}{|l|}{ SV involvement } \\
\hline No & 49 & $8.96(2.6)$ & 0.684 & $3.04(1.2)$ & 0.182 & $12.01(3.7)$ & 0.468 \\
\hline Yes & 11 & $9.30(2.5)$ & & $4.76(3.0)$ & & $12.29(3.3)$ & \\
\hline \multicolumn{8}{|l|}{ Margins Positive } \\
\hline No & 41 & $9.40(2.6)$ & 0.105 & $3.22(1.3)$ & 0.469 & $12.55(3.7)$ & 0.876 \\
\hline Yes & 21 & $8.25(2.5)$ & & $3.0(1.0)$ & & $11.25(3.4)$ & \\
\hline \multicolumn{8}{|l|}{ Extracapsular } \\
\hline No & 46 & $8.91(2.8)$ & 0.633 & $3.39(2.0)$ & 0.875 & 11.83 (3.8) & 0.471 \\
\hline Yes & 17 & $9.26(2.0)$ & & $3.31(0.9)$ & & $12.57(2.8)$ & \\
\hline \multicolumn{8}{|l|}{ Metastasis } \\
\hline No & 52 & $8.95(2.7)$ & 0.723 & $3.11(1.2)$ & 0.640 & 12.07 (3.7) & 0.682 \\
\hline Yes & 12 & $9.25(2.04)$ & & $4.50(3.1)$ & & $11.97(2.7)$ & \\
\hline
\end{tabular}

highlight the marked differences between patients with benign versus cancer. Adjusting this cut point for Hp1gamma overexpression can increase the sensitivity or specificity depending on its required use. Total expression was associated with higher Gleason score $(\mathrm{p}=0.04)$.

One notable finding was that $\mathrm{HP} 1 \gamma$ was not only an independent predictor of PSA-recurrence for patients who underwent radical prostatectomy, but was superior to pathological Gleason score using both a multivariate Cox model and a Kaplan-Meier analysis (Table 3 and Figure 4). Few biomarkers to date are able to predict PSA recurrence more robustly than Gleason score. Recently, Cuzick et al. reported a panel of 31 RNA markers had a more significant $P$ value compared to Gleason

Table 2 Univariate cox regression analysis for predicting biochemical recurrence

\begin{tabular}{lccccc}
\hline Variables* & Coefficent & s.e. & Wald & P & Odd ratio (95\% Cl) \\
\hline Gleason category & 0.392 & 0.197 & 4.025 & 0.045 & $1.485(1.009-2.186)$ \\
Stage (pT) category & 0.860 & 0.355 & 5.853 & 0.016 & $2.363(1.177-4.741)$ \\
Tumor volume & 0.051 & 0.298 & 0.029 & 0.864 & $1.052(0.587-1.887)$ \\
Seminal vesicle involvement & 1.195 & 0.542 & 4.860 & 0.027 & $3.305(1.142-9.566)$ \\
Margins & -0.154 & 0.574 & 0.072 & 0.789 & $0.858(0.278-2.641)$ \\
Extracapsular extension & 0.792 & 0.513 & 2.386 & 0.122 & $2.208(0.808-6.033)$ \\
Metastasis & 1.414 & 0.516 & 7.489 & 0.006 & $4.112(1.495-11.312)$ \\
Nuclear Hp1y (continuous) & 0.043 & 0.107 & 0.165 & 0.685 & $1.044(0.847-1.287)$ \\
Cytoplasmic Hp1y (continuous) & 0.751 & 0.356 & 4.449 & 0.035 & $2.118(1.055-4.255)$ \\
\hline
\end{tabular}


Table 3 Multivariate cox regression analysis for predicting biochemical recurrence

\begin{tabular}{lccccc}
\hline Variables* & Coefficent & s.e. & Wald & $P$ & Odd ratio (95\% Cl) \\
\hline Remained in model: $(P<0.05):$ & & & & & \\
Metastasis & 1.690 & 0.690 & 6.005 & 0.014 & $5.418(1.402-20.930)$ \\
Cytoplasmic Hp1Y (continuous) & 0.899 & 0.432 & 4.320 & 0.038 & $2.456(1.053-5.732)$ \\
\hline
\end{tabular}

*Variables removed from the model $(P>0.05)$ : Gleason category, stage (pT) category, seminal vesicle involvement.

score for predicting PSA recurrence [20]. One explanation for the robustness of $\mathrm{HP} 1 \gamma$ is that its prognostic value is not collinearly associated with other clinicopathologic variables (Table 1). Given the role $\mathrm{HP} 1 \gamma$ plays in gene repression $[3,21]$ and development [4], we postulate that aberrant expression of this protein may be central to the pathophysiology of PCa.

Increased expression of $\mathrm{HP} 1 \gamma$ in the nucleus compared to the cytoplasm is consistent with data demonstrating this protein is primarily localized to centromeric and telomeric heterochromatin $[3,8]$ and euchromatin $[22,23]$. It was of interest to find expression of the protein in the cytoplasm and note that this carried prognostic significance. Its presence in the cytoplasm may represent altered protein processing, or may have some as of yet unknown functional significance. Ki-67 is a nuclear protein that correlates with cell proliferation and is a known marker of $\mathrm{PCa}$ progression [24]. The Cterminal domain has been found to bind to all three members of the HP1 family [13]. We found a significant association between HP1 $\gamma$ and Ki-67 on a per core basis
(Figure 2). Given the more uniform expression of $\mathrm{HP} 1 \gamma$ across prostate cancer cells when compared to Ki-67, it clearly has other functions independent from Ki-67 in the regulation of heterochromatin.

\section{Conclusion}

In conclusion, we demonstrate that $\mathrm{HP} 1 \gamma$ expression is elevated in $\mathrm{PCa}$ and this independently predicts PSA-recurrence for patients more accurately than pathological Gleason score. Use of novel biomarkers to identify men at lower risk for recurrence may reduce the

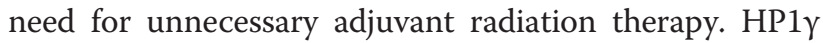
represents a novel marker of prostate cancer progression that will be useful to the clinician with further validation in other datasets. In addition, examination of HP1 $\gamma$ expression in biopsy specimens might fulfill an urgent need for more accurate pre-treatment risk stratification tools. Given the role $\mathrm{HP} 1 \gamma$ plays in epigenetic gene regulation through its binding to methylated lysine on histone $\mathrm{H}_{3}$ (H3K9me) [7], it represents a novel target for cancer treatment. Furthermore, a recent survey of
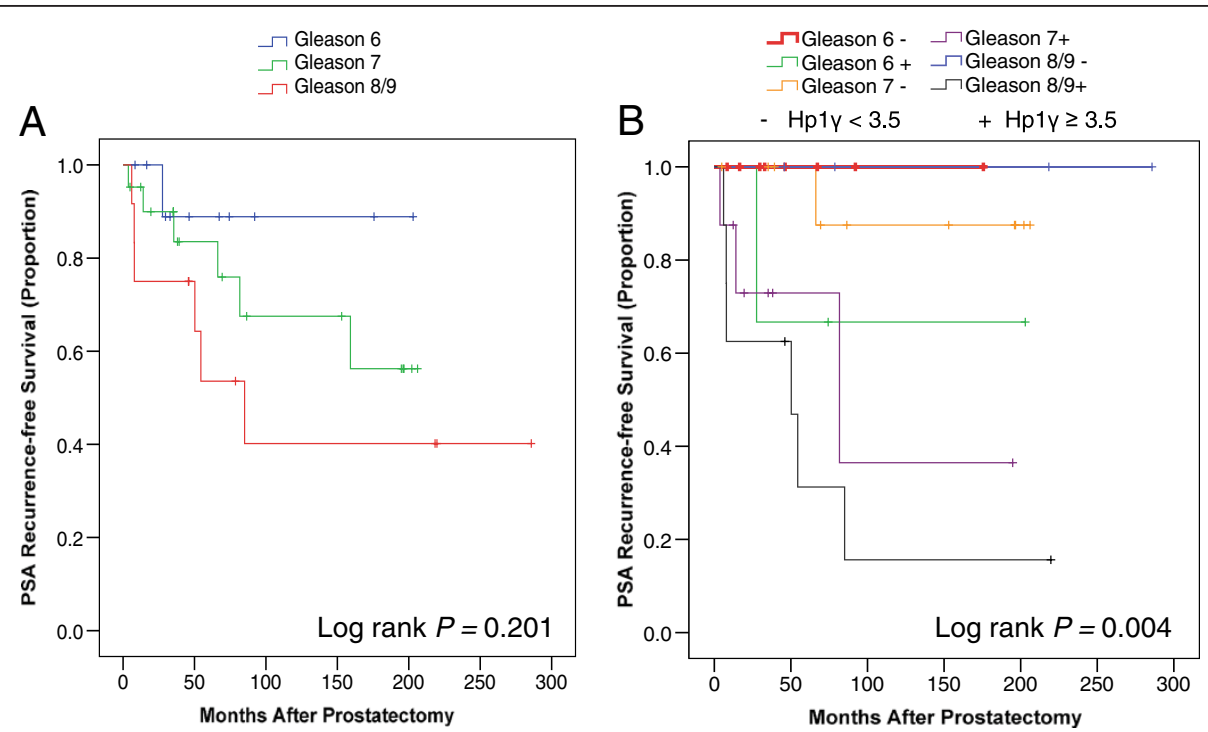

Figure 4 Kaplan Meier analysis comparing pathological Gleason score versus Hp1\}. PSA failure after radical prostatectomy was examined in samples stained for Hp1y. A) Gleason score provides minimal separation of patients into risk categories for biochemical recurrence using a Log rank analysis $(P=0.2)$. B) Cytoplasmic Hp1y is superior to pathological Gleason score when Gleason score was stratified into high versus low Hp1y. The median value for Hp1y staining in was 3.5 in all benign and cancer samples and this value was subsequently used as a cut point to define high versus low cytoplasmic Hp1y expression for the Log rank analysis. Hp1y accurately predict biochemical recurrence within each Gleason category including intermediate grade cancer (GS 6, 7). 
tumors suggests HP1 $\gamma$ may be overexpressed in other epithelial cancers [3].

\section{Abbreviations}

PCa: Prostate cancer; HP1Y: Heterochromatin protein $\gamma_{;}$H3K9me: Histone 3 lysine 9 methylated; TMA: Tissue microarray; GS: Gleason score.

\section{Competing interests}

The authors declare that they have no competing interests.

\section{Authors' contributions}

JS, MT, and DJ analyzed the results and wrote the paper. MT performed statistical analysis. WH carried out the experiments and operated VECTRA ${ }^{\text {TM }}$ technology. DJ designed the study and provided supervision. All authors read and approved the final manuscript.

\section{Acknowledgements}

This work was supported by the National Institutes of Health 5R01CA097131 [DJ], and JS was supported by the training grant T35 DK062709-06 (http:// grants.nih.gov/grants/). JS and MT were also supported by University of Wisconsin SMPH Shapiro grants.

\section{Author details}

'School of Medicine and Public Health, University of Wisconsin-Madison, Madison, WI 53750, USA. ${ }^{2}$ Department of Pathology and Laboratory Medicine, University of Wisconsin-Madison, Madison, WI 53750, USA. ${ }^{3}$ Department of Urology, University of Wisconsin-Madison, Madison, WI 53705, USA.

Received: 27 September 2012 Accepted: 18 March 2013 Published: 23 March 2013

\section{References}

1. Siegel R, Naishadham D, Jemal A: Cancer statistics, 2012. CA Cancer J Clin 2012, 62(1):10-29.

2. Schaeffer EM, Marchionni L, Huang Z, Simons B, Blackman A, Yu W, Parmigiani G, Berman DM: Androgen-induced programs for prostate epithelial growth and invasion arise in embryogenesis and are reactivated in cancer. Oncogene 2008, 27(57):7180-7191.

3. Dialynas GK, Vitalini MW, Wallrath LL: Linking Heterochromatin Protein 1 (HP1) to cancer progression. Mutat Res 2008, 647(1-2):13-20.

4. Shapiro E, Huang H, Ruoff R, Lee P, Tanese N, Logan SK: The heterochromatin protein 1 family is regulated in prostate development and cancer. J Urol 2008, 179(6):2435-2439.

5. Takanashi M, Oikawa K, Fujita K, Kudo M, Kinoshita M, Kuroda M: Heterochromatin protein 1gamma epigenetically regulates cell differentiation and exhibits potential as a therapeutic target for various types of cancers. Am J Pathol 2009, 174(1):309-316.

6. Caillier M, Thénot S, Tribollet V, Birot AM, Samarut J, Mey A: Role of the epigenetic regulator HP1 $y$ in the control of embryonic stem cell properties. PLoS One 2010, 5(11):e15507.

7. Lomberk $G$, Wallrath $L$, Urrutia R: The Heterochromatin Protein 1 family. Genome Biol 2006, 7(7):228.

8. Canudas S, Houghtaling BR, Bhanot M, Sasa G, Savage SA, Bertuch AA Smith S: A role for heterochromatin protein $1 \gamma$ at human telomeres. Genes Dev 2011, 25(17):1807-1819.

9. Zhang R, Liu ST, Chen W, Bonner M, Pehrson J, Yen TJ, Adams PD: HP1 proteins are essential for a dynamic nuclear response that rescues the function of perturbed heterochromatin in primary human cells. Mol Cell Biol 2007, 27(3):949-962.

10. Luijsterburg MS, Dinant C, Lans H, Stap J, Wiernasz E, Lagerwerf S, Warmerdam DO, Lindh M, Brink MC, Dobrucki JW, et al: Heterochromatin protein 1 is recruited to various types of DNA damage. J Cell Biol 2009, 185(4):577-586.

11. Tanaka M, Chang P, Li Y, Li D, Overman M, Maru DM, Sethi S, Phillips J, Bland GL, Abbruzzese JL, et al: Association of CHFR promoter methylation with disease recurrence in locally advanced colon cancer. Clin Cancer Res 2011, 17(13):4531-4540.

12. Kirschmann DA, Lininger RA, Gardner LM, Seftor EA, Odero VA, Ainsztein AM, Earnshaw WC, Wallrath LL, Hendrix MJ: Down-regulation of
HP1Hsalpha expression is associated with the metastatic phenotype in breast cancer. Cancer Res 2000, 60(13):3359-3363.

13. Scholzen T, Endl E, Wohlenberg C, van der Sar S, Cowell IG, Gerdes J, Singh PB: The Ki-67 protein interacts with members of the heterochromatin protein 1 (HP1) family: a potential role in the regulation of higher-order chromatin structure. J Pathol 2002, 196(2):135-144.

14. Huang W, Hennrick K, Sally Drew BS M: A colorful future of quantitative pathology: validation of Vectra technology using chromogenic multiplexed immunohistochemistry and prostate tissue microarrays. Hum Pathol 2012. in press.

15. Ogawa H, Ishiguro K, Gaubatz S, Livingston DM, Nakatani Y: A complex with chromatin modifiers that occupies E2F- and Myc-responsive genes in G0 cells. Science 2002, 296(5570):1132-1136.

16. Gerdes J, Schwab U, Lemke H, Stein H: Production of a mouse monoclonal antibody reactive with a human nuclear antigen associated with cell proliferation. Int J Cancer 1983, 31(1):13-20.

17. Gerdes J, Lemke H, Baisch H, Wacker HH, Schwab U, Stein H: Cell cycle analysis of a cell proliferation-associated human nuclear antigen defined by the monoclonal antibody Ki-67. J Immunol 1984, 133(4):1710-1715.

18. Chun FK, Briganti A, Graefen M, Porter C, Montorsi F, Haese A, Scattoni V, Borden L, Steuber T, Salonia A, et al: Development and external validation of an extended repeat biopsy nomogram. J Urol 2007, 177(2):510-515.

19. Veltri RW, Isharwal S, Miller MC, Epstein Jl, Mangold LA, Humphreys E, Partin AW: Long-term assessment of prostate cancer progression free survival: evaluation of pathological parameters, nuclear shape and molecular biomarkers of pathogenesis. Prostate 2008, 68(16):1806-1815.

20. Cuzick J, Swanson GP, Fisher G, Brothman AR, Berney DM, Reid JE, Mesher D, Speights VO, Stankiewicz E, Foster CS, et al: Prognostic value of an RNA expression signature derived from cell cycle proliferation genes in patients with prostate cancer: a retrospective study. Lancet Oncol 2011, 12(3):245-255.

21. Kamakaka RT: Heterochromatin: proteins in flux lead to stable repression. Curr Biol 2003, 13(8):R317-319.

22. Lomberk G, Bensi D, Fernandez-Zapico ME, Urrutia R: Evidence for the existence of an HP1-mediated subcode within the histone code. Nat Cell Biol 2006, 8(4):407-415

23. Minc E, Courvalin JC, Buendia B: HP1gamma associates with euchromatin and heterochromatin in mammalian nuclei and chromosomes. Cytogenet Cell Genet 2000, 90(3-4):279-284.

24. Bettencourt MC, Bauer JJ, Sesterhenn IA, Mostofi FK, McLeod DG, Moul JW: $\mathrm{Ki}-67$ expression is a prognostic marker of prostate cancer recurrence after radical prostatectomy. J Urol 1996, 156(3):1064-1068.

doi:10.1186/1471-2407-13-148

Cite this article as: Slezak et al: HP1 1 expression is elevated in prostate cancer and is superior to Gleason score as a predictor of biochemical recurrence after radical prostatectomy. BMC Cancer 2013 13:148.

\section{Submit your next manuscript to BioMed Central and take full advantage of:}

- Convenient online submission

- Thorough peer review

- No space constraints or color figure charges

- Immediate publication on acceptance

- Inclusion in PubMed, CAS, Scopus and Google Scholar

- Research which is freely available for redistribution 